\title{
Erratum to: Estimating trace deposition time with circadian biomarkers: a prospective and versatile tool for crime scene reconstruction
}

\author{
Katrin Ackermann • Kaye N. Ballantyne • \\ Manfred Kayser
}

Published online: 3 June 2010

(C) Springer-Verlag 2010

\section{Erratum to: Int J Legal Med \\ DOI 10.1007/s00414-010-0457-1}

The original version of this article, including the ESM, unfortunately contained a mistake.

The Figures 4, 5, and S6 (the latter in the ESM) of this article (stored blood and saliva samples) all contain the same

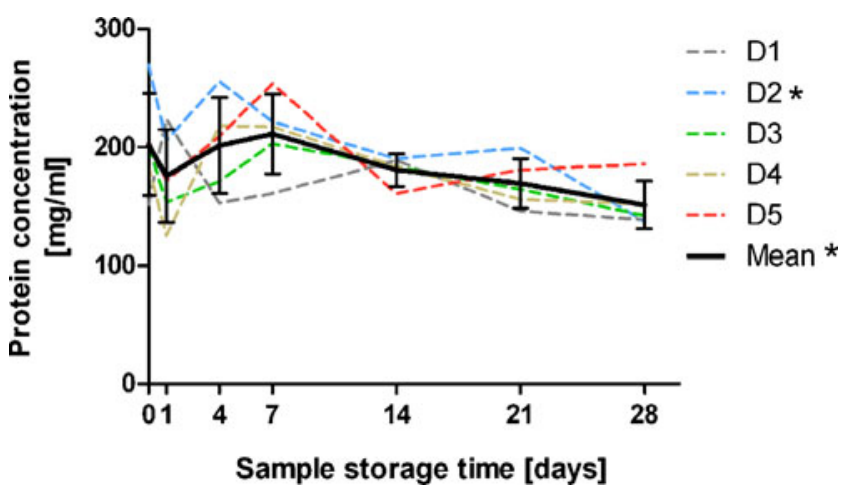

Fig. 4 Total protein concentrations obtained from five sets of dried blood stains in six time intervals over a period of 4 weeks, and the respective mean $( \pm \mathrm{SD})$ values; statistically significant decay of individual concentration values are indicated with $* p<0.05$; parafilm samples, D1 and D4; cotton samples, D2, D3, and D5

The online version of the original article can be found at http://dx.doi. org/10.1007/s00414-010-0457-1.

Electronic supplementary material The online version of this article (doi:10.1007/s00414-010-0471-3) contains supplementary material, which is available to authorized users.

K. Ackermann $\cdot$ K. N. Ballantyne $\cdot$ M. Kayser $(\bowtie)$

Department of Forensic Molecular Biology,

Erasmus University Medical Center Rotterdam,

PO Box 2040, 3000 CA Rotterdam, Netherlands

e-mail: m.kayser@erasmusmc.nl

URL: www.erasmusmc.nl/fmb/ error that occurred during the plotting of the data points (data points were shown equally spaced). The corrected figures with the appropriate $x$ axes are provided below (and in the corrected ESM). As a result, the linear regression analysis for some of the data sets changed as indicated in the figures. However, these corrections do not affect any of the conclusions of the work.

The correct version of the ESM is shown at ESM Fig. S6.
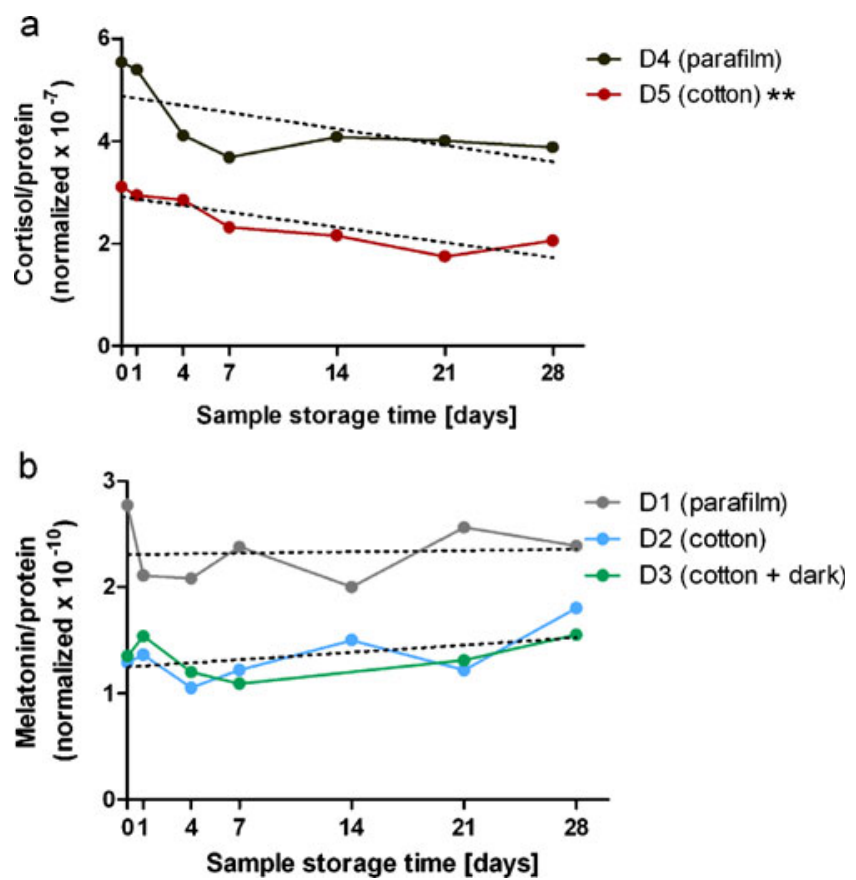

Fig. 5 a Normalized cortisol concentrations obtained from two individual data sets of dried blood stains over a period of 4 weeks including the respective linear regression lines fitting the data points; statistically significant decay of individual concentration values are indicated with $* * p=0.009$. b Normalized melatonin concentrations obtained from three individual sets of dried blood stains over a period of 4 weeks, including the respective linear regression lines fitting the data points for set D1 and sets D2/D3; none of the sets showed significant linear relationships with storage time 